\title{
When Wide Scope Is not Enough: Scope and Topicality of Discourse Referents
}

\author{
Gemma Barberà \\ Universitat Pompeu Fabra \\ Roc Boronat, 138, Barcelona, Spain \\ gemma.barbera@upf.edu \\ http://parles.upf .edu/llocs/gbarbera/
}

\begin{abstract}
This paper analyses the semantic attributes discourse referents in Catalan Sign Language may have in order to have a corresponding location established in sign space. It is argued that a combination of scope and topicality is required when analysing the correlation between the introduction of entities into the discourse and assigning a spatial location.
\end{abstract}

Keywords: Catalan Sign Language (LSC), location, modal subordination, scope, sign space, specificity

\section{Introduction}

As natural languages in a visual-spatial modality, sign languages use the space in front of the signer's torso to articulate signs. Sign space is morphosyntactically relevant, since signs are spatially modulated for grammatical purposes to express number, person, and arguments of the verb. It is also relevant at the discourse level because it is commonly assumed that entities introduced into the discourse model are identified with certain spatial locations established on the horizontal plane [12], which is the plane that extends parallel to the floor [2]. However, not all the entities introduced have a corresponding spatial location and the semantic attributes that discourse referents should have in order to be spatially localised have not been thoroughly analysed. Moreover, the frontal plane, which extends parallel to the signer's body, has not been analysed when considering the spatial establishment of entities.

Catalan Sign Language (LSC) makes systematic use of signs directed to the horizontal plane, as commonly assumed for other sign languages (SLs) but also to the frontal plane. This paper focuses on the grammatical distinction denoted by the establishment of discourse referents within the two parts of the frontal plane, namely upper and lower. It is argued that this distinction is relevant for LSC grammar and it is explained in terms of scope behavior as well as topicality. My main claims are interrelated: (i) The expression of narrow scope quantifiers leads to a lack of spatial location establishment; however when focusing on specificity contexts, (ii) narrow scope related to specificity is overtly encoded in LSC 
grammar and, more particularly, it is expressed with marked spatial locations established on the upper part of the frontal plane; yet (iii) narrow scope variables can also establish a lower spatial location as long as they denote a prominent discourse referent. Although claim (ii) and (iii) may seem to be contradictory, I show that they are in fact complementary once discourse structure is included in the analysis.

The rest of the paper is structured as follows. $\S 2$ analyses the relation between dependent variables and sign space in LSC. $\S 3$ focuses on specificity marking. $\S 4$ presents contexts of modal subordination where variables attached to narrow scope quantifiers discursively behave as wide scope ones. $§ 5$ presents the interaction between scope and prominence, and $\S 6$ concludes.

\section{Dependent variables}

Discourse referents (DRs) are semantic objects which denote the object of thought or the thing the discourse is about. Once established in the discourse they can be referred back to by a pronoun or retrieved by a definite description [11]. In dynamic semantics, variables are the construct which correspond to DRs. Inspired by [6], dependent variables are introduced into the model the values assigned to which co-vary with those assigned to another variable. Here I consider contexts where a universal quantifier or an operator binds the variable. Classical Discourse Representation Theory [10] considers that donkey sentences include universal quantification which takes scope over the entire sentence, and unselectively binds all the free variables in it. In LSC only DRs attached to wide scope quantifiers are spatially localised. The expression of narrow scope quantifiers leads to the lack of establishment of a spatial location, as contexts of dependent variables such as donkey sentences, genericity and quantified noun phrases (NPs) show.

In LSC donkey sentences, ${ }^{1}$ nominals do not occur with a determiner index sign directed to space to establish a location, but rather are uttered as bare nouns and hardly ever localised. As shown by [15], verb agreement is realised in a neutral location (1). ${ }^{2}$

IF TOWN FARMER HORSE THERE-IS, SURE 1-TAKE-CARE- ${ }_{c}$. 'If a farmer owns a horse, he certainly takes care of it'.

Correspondingly, in the DRT semantic representation of (1) the variable is represented under an embedded context.

\footnotetext{
${ }^{1}$ Cf. [18] for an analysis of donkey sentences in American SL and French SL, where it is argued in line with [13] that SL variables are overtly expressed.

${ }^{2}$ Glossing conventions: Manual signs are represented by the capitalised word corresponding to the translation of the sign; IX3 (pointing sign directed to the lateral parts of space); \#-VERB-\# (verb agreeing with subject and object: the numbers refer to the grammatical person); subindices mark direction towards space: 1 (low), u (up), ip (ipsilateral) cl (contralateral), ce (centre); +++ (reduplication of signs).
} 
$(2)$

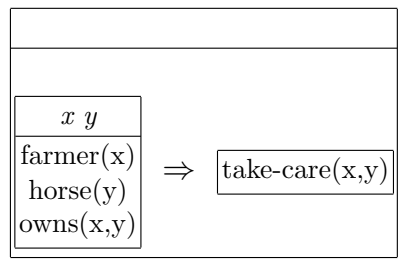

Another context of a dependent variable is that of genericity. Generic statements express general claims about kinds, rather than claims about particular entities. In LSC bare nouns assume a generic interpretation when they are not localised [14], as shown in (3). Any attempt to localise the DR in space is understood as referential, i.e. as denoting a specific man (5). Generic statements are represented according to the idea that a generic operator binds particular variables in its scope. As shown in the corresponding DRS of (3), variables appear in the complex construction represented by a subordinate DRS bound by the generic operator (4).

$(3)$

$$
\text { MAN PLAY LIKE }
$$

'Men like to play'

(4)

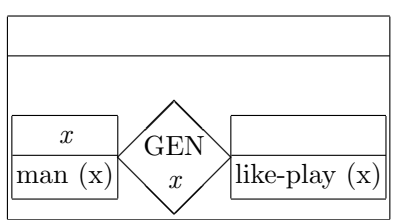

$(5)$

(6)

MAN IX $3_{i p}$ PLAY LIKE 'A/the man likes to play'

\begin{tabular}{|c|}
\hline$x$ \\
\hline man $(\mathrm{x})$ \\
like-play (x) \\
\hline
\end{tabular}

The third argument comes from quantified NPs. In American SL quantified expressions do not establish a spatial location, and sign space is only used to quantify over the domain [12]. In LSC the verbal morphology influences the quantificational interpretation of the bare noun STUDENT [14], as shown in (7). In the corresponding DRS the variable is embedded under the scope of the quantifier (8).

$$
\text { STUDENT EACH-ONE+++ TEACHER ASK }+++
$$
'Each pupil asked his/her teacher.'

(8)

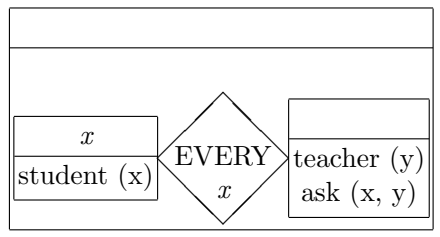

As seen in these examples, when the variable is bound there is a lack of spatial location establishment in LSC. Hence, spatial locations can be defined as the overt manifestation of the DR attached to a quantifier that has wide scope only. Nevertheless, narrow scope does not entail a lack of spatial location establishment but rather a marked location is established on the upper part of the frontal plane for non-specific DRs. 


\section{$3 \quad$ Specificity marking}

Whether definiteness is grammatically encoded in SLs is still a matter of debate among SL linguists. While some works argue that an index sign directed to space is the formal marking of definiteness [1], other works have questioned the definiteness marking of index signs [5]. In contrast, in LSC there is no formal marking to distinguish (in)definiteness. As shown in (9), an NP co-occurring with an index sign directed to space is ambiguous between having a definite or an indefinite interpretation.

\section{(9) TODAY IX1 INTERVIEW IX $3_{i p}$ WOMAN.}

a. Today I have an interview with a woman.

b. Today I have an interview with the woman.

In LSC the localisation of indefinite NPs spatially differs according to the specificity interpretation. Signs can be localised on the lower and the upper part of the frontal plane and this distinction corresponds to the overt marking of specificity (10) and non-specificity (11), respectively.

\section{IX1 INTERVIEW IX $3_{l}$ WOMAN}

I have an interview with a woman $_{\text {spec }}$

\section{IX1 INTERVIEW IX $3_{u}$ WOMAN}

I have an interview with a woman ${ }_{\text {nonspec }}$

The properties specificity encompasses, namely scope and partitivity, can be distinguished in the two localisation processes towards the frontal plane. Scopal specificity is defined in terms of the interpretation of the indefinite NP outside the scope of an operator. According to this view specificity is equated with wide scope $[6,8,9]$. Hence indefinite NPs which are outside the scope of an operator are considered to have wide scope, and indefinite NPs under the scope of an operator are treated as narrow scope ones.

In LSC indefinite NPs are not ambiguous between having a specific or a nonspecific reading. Specific NPs are established on the lower frontal plane (12), whereas non-specific NPs are established on the upper part (13). ${ }^{3}$

I want to buy a cat. It is very obedient.

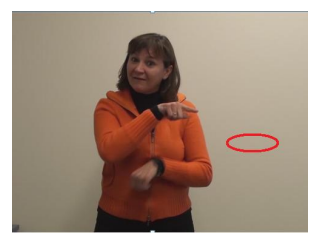

I want to buy a cat. It must be obedient.

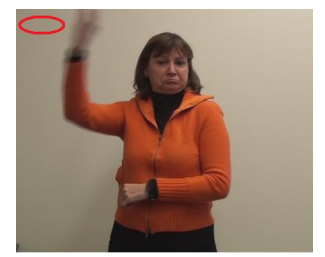

\footnotetext{
${ }^{3}$ For the interest of space, these examples are provided with the English counterpart of the LSC sentence. The NP localised in space shown in the still is marked with boldface.
} 
The implementation of specificity marking is formally represented with a variable appearing in the main DRS. This variable has wide scope over the other possible embedded variables in the subordinated DRS (14). Non-specificity is implemented with a subordinate variable embedded under the modality operator $(15)$.

$$
\begin{array}{|c|}
\hline x y \\
\hline \text { cat }(\mathrm{x}) \\
\square \text { buy }(1, \mathrm{x}) \\
\hline \text { it }(\mathrm{y}) \\
\text { obedient }(\mathrm{y}) \\
\mathrm{y}=\mathrm{x} \\
\hline
\end{array}
$$

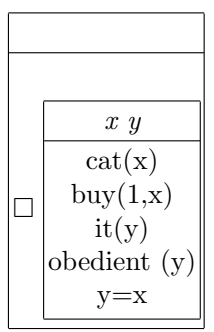

The other specificity property considered in the present analysis is partitivity. Partitive indefinite NPs receive a semantic partitive interpretation when the denotation of the NP is included within a given set and they have a restricted set as a possible value. The quantification ranges over some specific, non-empty, contextually fixed set. [4] views specificity as partitivity, since in Turkish NPs ambiguity is resolved through case marking. NPs with overt case morphology are partitive and they introduce into the domain of discourse entities from a previously given set. Partitive NPs denote a specific DR. In contrast, NPs without case morphology are non-partitive which denote a non-specific DR.

In LSC there is a difference between NPs which have a restriction of the quantified NP and those which do not have such a restriction. This is marked in LSC with a difference on the two opposed directions on the frontal plane [15]. Under the restriction of the quantified NP, LSC locations are established on the lower frontal plane (16). When there is no such restriction, the upper frontal plane is used (17), as shown in the LSC counterparts below.

Some of the friends were hidden there for two years.

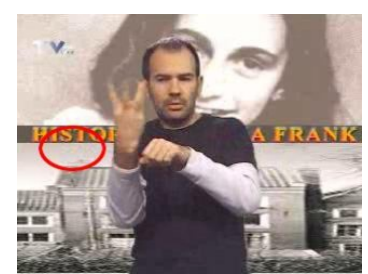

Someone denounced they were there.

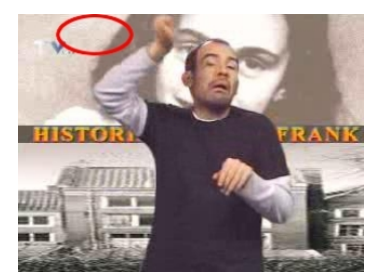

The quantifier in (16) is an element of the group denoted by the NP. This is shown in the corresponding DRS by the relation $\mathrm{x} \in \mathrm{X}$, where $\mathrm{X}$ corresponds to a non-atomic variable that is projected to the main universe. $\mathrm{x}$ is an atomic variable and it is a subset of X. Although $\mathrm{x}$ is not projected into the main DRS, it belongs to the set (18). In contrast, the sentence in (17) denotes a non-specific DR which does not belong to a contextually determined set. In the corresponding 
DRS, this is represented with an embedded variable which does not belong to any set from the main DRS (19).

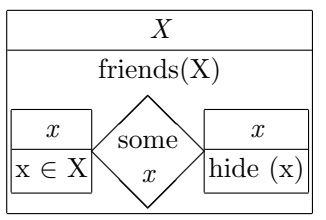

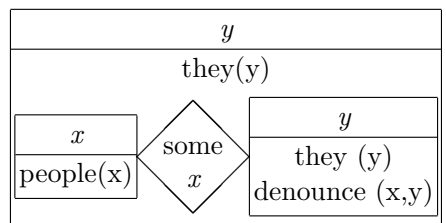

Previously, in $\S 2$ it has been shown that when the variable is bound there is a lack of spatial location establishment. However, this section has shown that when considering specificity marking, binding of an operator can also establish a marked location on the upper frontal plane. Upper locations denote that there is no restriction of the quantified NP and they occur with scopally non-specific DRs.

\section{Modal subordination}

This section is devoted to the analysis of narrow scope variables which behave as wide scope ones. Here I focus on modal subordination contexts which consist of noteworthy DRs which are introduced into the model and the existence of which is not presupposed. Modal subordination are anaphoric contexts which are under the scope of a modal operator or a propositional attitude predicate, but display anaphoric relations that appear at first glance to violate generalisations about scope operators and anaphoric potential [17].

In LSC modal subordination contexts, the variable is attached to a narrow scope quantifier but behaves as a wide scope variable. This behaviour is overtly expressed in LSC with localisation of signs. In (20) the DR 'person' refers to a non-specific and non-identifiable entity. As indicated in the subindices, it is localised towards the upper part of the frontal plane.

$$
\begin{aligned}
& \text { IX1 THINK IX3 BOOK 1-OFFER- } 3_{c l-u} \text { ADEQUATE PERSON- } 3_{u} \ldots \\
& \text { MUST PERSON- } 3_{c-u} \text { LIKE HOBBY } \\
& \text { PAST SAME/ALWAYS. } \\
& \text { IX } 3_{i p-l} \text { IX1 } 1 \text {-OFFER- } 3_{i p-l} \text { PERSON- } 3_{i p-l} \text { IX } 3_{i p-l} \\
& \text { 'I think that I would offer this book to a person }{ }_{\text {nonspec }} \ldots \\
& \text { It must be someone who likes traditional things. } \\
& \text { Definitely, I would offer it to him/her.' }
\end{aligned}
$$

While introducing the antecedent ('someone who likes traditional things') the signer directs a darting eyegaze to an upper direction that goes from the ipsilateral, center and to the contralateral part (21). This eyegaze moves around along the upper frontal plane, without being directly fixed to an area. It functions as an overt operator denoting a de dicto mode. Once the intensional context is established by this de dicto mode, every subsequent sentence is anchored to 
this mode even across sentence boundaries and all the variables in the semantic representation are bound by the operator.

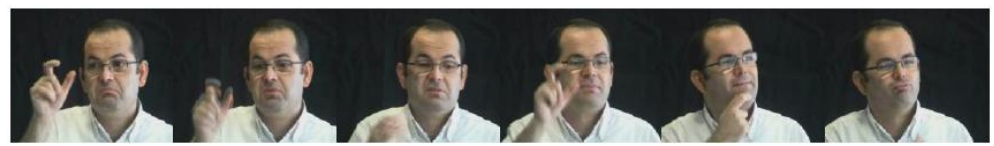

However, as shown in the third utterance in (20) the signer directs pronominal signs and an agreement verb towards a lower spatial location. Hence in subsequent sentences resumptive pronouns referring back to an antecedent which is bound by an operator may also establish a lower spatial location. As shown in (22), both antecedent and consequent are bound by the necessity operator. As long as the variable is under the scope of the corresponding operator, resumptive pronouns are thus felicitous.

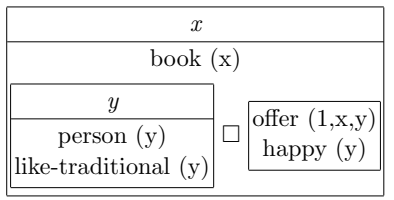

In LSC modal subordination contexts, a lower spatial location is established for a narrow scope variable once the intensional context has been set. Although modal subordination contexts seem to contradict the hypothesis presented in $\S 3$ where narrow scope variables have been analysed as being overtly expressed on the upper frontal plane, this apparent puzzle is resolved once we introduce the discursive notion of 'prominence'. Hence when studying the establishment of DRs in sign space, the analysis in terms of scope is not enough, but discourse structure and topicality of entities must also be incorporated.

\section{Prominent narrow scope variables}

Prominence is defined as the degree of relative salience of a unit of information, at a specific point in time, in comparison to the other units of information [3]. Centering Theory represents probably the most influential account of entity-based prominence in discourse [7]. This processing model relates the local utteranceby-utterance context and discourse anaphoric reference. It constitutes a basis to theorise about local coherence, prominence and choice of referring expressions. Centering has a set of basic notions, which are defined and adapted to the present account in what follows. A discourse model contains:

(i) a set of forward looking DRs (henceforth, $\operatorname{DRf}\left(\mathrm{U}_{k}\right)$ ), which appear in the DRS of $\mathrm{K}$ and that can be referred to in subsequent utterances;

(ii) a backward looking $\operatorname{DR}\left(\operatorname{DRb}\left(\mathrm{U}_{k}\right)\right)$, which is a unique entity defined for each utterance $\mathrm{U}_{k}$ (except for the intial segment) that refers back to a forward looking DR of the preceding utterance $\mathrm{U}_{k-1}$, and that intuitively represents the $\mathrm{DR}$ which is the center of attention at utterance $\mathrm{U}_{k}$; 
(iii) a preferred $\operatorname{DR}\left(\operatorname{DRp}\left(\mathrm{U}_{k}\right)\right)$, which is the one that is on the top of the hierarchy of the set of DRs in the main DRS.

Although in most cases topics tend to represent old information, this is neither a sufficient nor a necessary condition for topicality. Topics are better analysed in terms of their effect on the ongoing discourse and considering the effects of previous discourse on the given utterance, rather than as old information $[16,19]$. A DR is linked to the discourse topic of the fragment of discourse (i.e. it is the most prominent entity of that specific fragment) if it verifies the following formula:

$$
\operatorname{DRb}\left(\mathrm{K}_{n}\right)=\operatorname{DRb}\left(\mathrm{K}_{n-1}\right) \wedge \operatorname{DRb}\left(\mathrm{K}_{n}\right)=\operatorname{DRp}\left(\mathrm{K}_{n}\right)
$$

Topicality is thus here verified when the variable corresponds to the intersection between the DR relations in previous utterances, as specified in the first argument of the formula, and the DR relations in subsequent utterances, as specified in the second argument. More concretely, in (23) the corresponding variable is the one that intersects between the backwards DR of the current utterance being the same as the backwards DR of the preceding utterance, and the backwards DR of the current utterance being the same as the preferred DR of the current utterance.

The variable which verifies the formula in (23) will be connected to the discourse topic and will be thus the most prominent DR at a specific point in discourse. The set of forward looking variables $\operatorname{DRf}\left(\mathrm{U}_{k}\right)$ are not only restricted to the ones appearing on the main DRS of $\mathrm{U}_{k}$, but also to subordinated variables as long as they are embedded under the corresponding operator. In LSC, the $\operatorname{DRb}\left(\mathrm{U}_{k}\right)$ among the $\operatorname{DRf}\left(\mathrm{U}_{k}\right)$ will be correlated with a lower spatial location as long as it verifies (23) and independently of the scope of the quantifier attached to the variable. This explains why modal subordination contexts in LSC although referring to non-specific DRs establish a lower spatial location. An example of a narrow scope variable which is prominent at a specific fragment of discourse is shown below.

I would offer the book to someone who likes traditional things. He would be very happy, and he would enjoy it a lot.

In (24) the variable that verifies (23) is z, as shown in (25). In the corresponding DRS a subindex $\mathrm{p}$ is assigned to the most prominent variable in the specific fragment of discourse.

$$
\left[\operatorname{DRb}\left(\mathrm{K}_{n}\right)=\operatorname{DRb}\left(\mathrm{K}_{n-1}\right) \wedge \operatorname{DRb}\left(\mathrm{K}_{n}\right)=\operatorname{DRp}\left(\mathrm{K}_{n}\right)\right] \equiv \mathrm{z}
$$




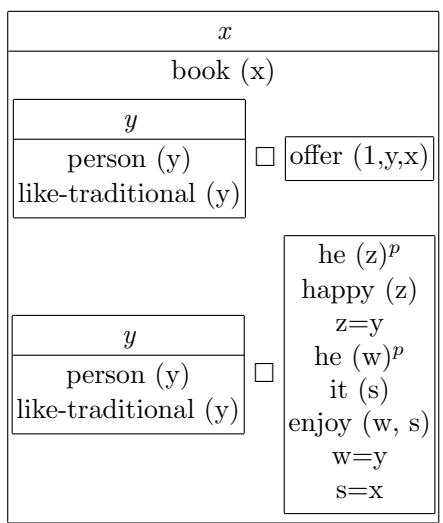

Both narrow and wide scope quantifiers attached to variables can be linked to the discourse topic and hence represent the most prominent DR. The assignment of the subindex into the corresponding variable allows to have the semantic representation for a fragment of discourse linked to its prominent structure. When the subindex is assigned, a lower spatial location in LSC sign space is established.

\section{Conclusions}

The proposal offered has determined the properties that DRs may have, which lead to the establishment of a location in sign space. It has offered a novel dynamic semantics account, which previous non-dynamic analyses have not been able to propose. Also a representational semantic level which integrates a theory of discourse structure with special focus on prominence has been offered. It has shown that the binding of an operator leads to a lack of spatial location establishment. Nevertheless, narrow scope does not entail the lack of spatial location establishment, but rather a marked location is established on the upper part of the frontal plane for non-specific DRs. LSC has an overt marking of specificity on the two parts of the frontal plane. But scope is not enough when studying the semantic attributes DRs need in order to have a corresponding spatial location in space, since the scope of the quantifier attached to the variable must be combined with the prominence of the variable at a point in the discourse. In cases of narrow scope marking the variable can establish a lower spatial location as long as it is connected to the prominent DR. In the future, cases of intermediate scope will be incorporated in the specificity analysis, as well as a refinement of the hierarchy motivations of prominence.

Acknowledgments. I am grateful to Josep Quer, Berit Gehrke and the audience at FEAST-Venice and at 18th Amsterdam Colloquium for interesting comments. Also my deaf colleagues Santiago Frigola and Delfina Aliaga deserve special credit for stimulating discussions. This research was partly made possible thanks to the Spanish Ministry of Science and Innovation (FFI2009-10492), 
Generalitat de Catalunya (URLING-2009SGR00763) and SignGram Cost Action IS1006. Of course, the remaining errors are all mine.

\section{References}

1. Bahan, B., Kegl, J., MacLaughlin, D., Neidle, C.: Convergent evidence for the structure of determiner phrases in American Sign Language. In: Gabriele, L., Hardison, D., Westmoreland, R. (eds.) FLSMVI. Proceedings of the Sixth Annual Meeting of the Formal Linguistics Society of Mid-America. Volume II: Syntax \& Semantics/Pragmatics. Indiana University Linguistics Club (1995)

2. Brentari, D.: A prosodic model of sign language phonology. The MIT Press, Cambridge, MA (1998)

3. Chiarcos, C., Claus, B., Grabski, M.: Salience in linguistisc and beyond. In: C. Chiarcos, B.C., Grabski, M. (eds.) Salience. Multidisciplinary perspectives on its function in discourse. Mouton de Gruyter, Berlin (2010)

4. Enç, M.: The semantics of specificity. Linguistic Inquiry 22(1), 1-25 (1991)

5. Engberg-Pedersen, E.: Space in Danish Sign Language: The semantics and morphosyntax of the use of space in a visual language. Signum Press, Hamburg (1993)

6. Farkas, D.F.: Dependent indefinites. In: F. Corblin, D.G., Marandin, J. (eds.) Empirical Issues in Formal Syntax and Semantics, pp. 243-267. Peter Lang Publishers (1997)

7. Grosz, B., Joshi, A., Weinstein, S.: Centering: A framework for modelling the local coherence of discourse. Computational Linguistics 2(21), 203-225 (1995)

8. von Heusinger, K.: Specificity and definiteness in sentence and discourse structure. Journal of Semantics 19, 245-274 (2002)

9. Ionin, T.: This is definitely specific: Specificity and definiteness in article systems. Natural Language Semantics 14, 175-234 (2006)

10. Kamp, H., Reyle, U.: From discourse to logic. Introduction to modeltheoretic semantics of natural language, formal logic and discourse representation theory. Kluwer Academic Press, Dordrecht (1993)

11. Karttunen, L.: Discourse referents. In: McCawley, J. (ed.) Syntax and Semantics: Notes from the Linguistic Underground, pp. 363-386. Academic Press, New York (1976)

12. Klima, E., Bellugi, U.: The signs of language. Harvard University Press, Cambridge, MA (1979)

13. Lillo-Martin, D., Klima, E.: Pointing out differences: ASL pronouns in syntactic theory. In: Fischer, S., Siple, P. (eds.) Theoretical Issues in Sign Language Research, Vol. 1: Linguistics, pp. 191-210. University Chicago Press, Chicago (1990)

14. Quer, J.: Quantifying across language modalities: generalized tripartite structures in signed languages (2005), presentation at the I Workshop on Sign Languages, EHU Vitoria-Gasteiz

15. Quer, J.: Signed agreement: Putting some more arguments together (2010), presentation at TISLR10. Purdue University, Indiana

16. Reinhart, T.: Pragmatics and linguistics. an analysis of sentence topics. Philosophica 27, 53-94 (1981)

17. Roberts, C.: Modal subordination and pronominal anaphora in discourse. Linguistics and Philosophy 12, 683-721 (1989)

18. Schlenker, P.: Donkey anaphora: the view from sign language (ASL and LSF). Linguistics \& Philosophy (to appear)

19. Vallduví, E.: The Informational component. Garland Press, New York (1992) 